The writer wishes to express his indebtedness to Professor C. W. Nelson of the University of California, Berkeley, who has checked the numerical tables.

Chin-Bing Ling

Aeronautical Research Lab.

and Academia Sinica

Taiwan, China

1. R. C. J. Howland, "On the stresses in the neighbourhood of a circular hole in a strip under tension," Roy. Soc., Phil. Trans., v. 229, Ser. A, 1930, p. 49-86.

2. R. C. J. Howland \& A. C. STEvenson, "Biharmonic analysis in a perforated strip," Roy. Soc., Phil. Trans., v. 232, Ser. A, 1934, p. 155-222.

3. C. B. LiNG, "Stresses in a notched strip under tension," Jn. Appl. Mech., v. 14, 1947, p. A275-280.

4. C. B. LiNG, "On the stresses in a notched strip," Jn. Appl. Mech., v. 19, 1952, p. 141-146.

5. C. B. LING, "Stresses in a perforated strip," Jn. Appl. Mech. (in press).

6. C. B. LING \& C. W. NeLSON, "On evaluation of Howland's integrals," Annals of Academia Sinica, Taiwan, China, v. 2, part 2, 1955, p. 45-50.

7. C. W. NELSON, "A Fourier integral solution for the plane-stress problem of a circular ring with concentrated radial loads," Jn. Appl. Mech., v. 18, 1951, p. 173-182.

8. J. W. L. GlaisheR, "Tables of $1 \pm 2^{-n}+3^{-n} \pm 4^{-n}$, etc., and $1+3^{-n}+5^{-n}+7^{-n}$ etc., to 32 places of decimals," Quart. Jn. of Math., v. 45, 1914, p. 141-158. The table also appears in H. T. Davis, Tables of Higher Mathematical Functions, v. 2, Principia Press, Bloomington, Indiana, 1955 .

9. Konrad Knopp, Infinite Series, Hafner Pub. Co., Inc., New York, 1947, p. 247.

10. J. W. L. GLAISHER, "Numerical values of the series $1-1 / 3^{n}+1 / 5^{n}-1 / 7^{n}+1 / 9^{n}-\ldots$, Messenger of Mathematics, v. 42, 1912, p. 35-49.

\title{
Tables for the Rapid and Accurate Numerical Evaluation of Certain Infinite Integrals Involving Bessel Functions
}

Introduction. In a recent paper [1] the author has formulated a method based on Euler's transformation of slowly convergent alternating series for the numerical evaluation of integrals of the form $\int_{a}^{\infty} f(x) d x$, where $a$ is a constant and where $f(x)$ oscillates about zero in such a way that the integral over each half-cycle is smaller in absolute magnitude than (and opposite in sign to) that over the preceding half-cycle. The author has had occasion to make much use of this method for the evaluation of integrals of the type

$$
\int_{0}^{\infty} J_{0}(x) g(x) d x
$$

and

$$
\int_{0}^{\infty} J_{1}(x) h(x) d x
$$

where $g(x), h(x)$ are well-behaved continuous functions which tend to a finite constant value or zero as $x$ tends to infinity; here $J_{0}(x), J_{1}(x)$ are Bessel Functions (of the first kind) of orders zero and one, respectively. The present paper gives tables useful in the evaluation of (1), (2).

The author is grateful to Yigal Accad who performed most of the numerical calculations for Tables 1 and 2 . 
Description of the Method. The method employed [1] in evaluating integrals of the type (1) involved performing the integration over each of the first twenty half-cycles, i.e., evaluating the integrals

$$
\int_{x_{i-1}}^{x_{i}} J_{0}(x) g(x) d x \quad i=1,2, \cdots, 20
$$

where $x_{0}$ is zero and $x_{i}$ is the $i$ th zero of $J_{0}(x)$. The first twenty terms of a slowly convergent alternating series for (1) were thus obtained. Euler's transformation (Bromwich [2], p. 62) was then applied to this series in order to obtain a rapidly convergent series for the numerical value of (1). Integrals of the type (2) were treated similarly.

In order to obtain high accuracy in the separate integrations (3) the Gauss quadrature formula (NBS AMS 37 [3]) was used for sixteen points of subdivision of each interval. Tables of abscissae and coefficients are given in [3] for $n=2(1) 16,15 \mathrm{D}$, and in Davis and Rabinowitz [7] for $n=2,4,8,16,20$, $24,32,40,48,20 \mathrm{D}$. Values are also available in $[7]$ for $n=64,80$ and 96 . For a given value of $n$, Gauss' formula provides an approximation equivalent to replacing the integrand by a polynomial of degree $2 n-1$.

Description and Use of the Tables. Table 1 gives values of $x$ and the corresponding values of $J_{0}(x)$. The values of $x$ were obtained by computation from values given in $[3]$ for the interval $(-1,1)$ by means of the formula

$$
x_{i}=x_{i}{ }^{1} \frac{q-p}{2}+\frac{q+p}{2}
$$

where $p, q$ are the lower and upper limits of integration, and in our case are zeros of $J_{0}(x)$ (the first value of $p$ is zero), and the $x_{i}{ }^{1}$ (given in [3]) refer to the interval $(-1,1)$. The zeros of $J_{0}(x)$ are also given.

The values of $J_{0}(x)$ were obtained by interpolation from the Harvard University tables [4]. In view of the high accuracy needed interpolation was effected by means of the first four terms of Taylor's theorem

$$
\begin{aligned}
J_{0}(x+h) \doteqdot & J_{0}(x)+h J_{0}^{\prime}(x)+\frac{h^{2}}{2 !} J_{0}^{\prime \prime}(x)+\frac{h^{3}}{3 !} J_{0}^{\prime \prime \prime}(x) \\
= & J_{0}(x)-h J_{1}(x)+\frac{1}{2} h^{2}\left(\frac{J_{1}(x)}{x}-J_{0}(x)\right) \\
& +\frac{1}{6} h^{3}\left(J_{1}(x)+\frac{J_{0}(x)}{x}-\frac{2 J_{1}(x)}{x^{2}}\right),
\end{aligned}
$$

these terms being sufficient for 10 place accuracy with the maximum value of $h$ required. The tables are believed to be accurate to within a few units in the tenth place of decimals.

Table 2 was similarly prepared for $J_{1}(x)$. Here the interpolation for $J_{1}(x)$ 
was effected from [4] by means of the Taylor's theorem expansion

$$
\begin{aligned}
J_{1}(x+h) \doteqdot J_{1}(x)+h\left(J_{0}(x)-\right. & \left.\frac{J_{1}(x)}{x}\right)+\frac{1}{2} h^{2}\left(\frac{2 J_{1}(x)}{x^{2}}-\frac{J_{0}(x)}{x}-J_{1}(x)\right) \\
& +\frac{1}{6} h^{3}\left(\frac{3 J_{0}(x)}{x^{2}}-\frac{6 J_{1}(x)}{x^{3}}+\frac{2 J_{1}(x)}{x}-J_{0}(x)\right) .
\end{aligned}
$$

In Tables 1 and 2 the zeros of $J_{0}(x), J_{1}(x)$ were taken from tables in BAASMTC [5].

Table 3 gives the appropriate integration coefficients (these are quoted from

\section{TABLE 3. Gauss Integration Coefficients}

$\begin{array}{ll}.02715 & 24594 \\ .06225 & 35239 \\ .09515 & 85117 \\ .12462 & 89713 \\ .14959 & 59888 \\ .16915 & 65194 \\ .18260 & 34150 \\ .18945 & 06105 \\ .18945 & 06105 \\ .18260 & 34150 \\ .16915 & 65194 \\ .14959 & 59888 \\ .12462 & 89713 \\ .09515 & 85117 \\ .06225 & 35239 \\ .02715 & 24594\end{array}$

[3] for the interval $(-1,1))$. After using them the result must be multiplied by half the length of the interval in each case.

Method of Checking. The tables were checked as follows:

Table 1 was checked by using it to calculate

$$
\int_{x_{i-1}}^{x_{i}} x J_{0}(x) d x
$$

over each half-cycle and comparing the result with the known value of the integral

$$
\left[x J_{1}(x)\right]_{x_{i-1}}^{x_{i}}=x_{i} J_{1}\left(x_{i}\right)-x_{i-1} J_{1}\left(x_{i-1}\right),
$$

the values of $J_{1}\left(x_{i}\right)$ being obtained from [5]. In each case agreement was obtained to within the accuracy warranted by the number of significant figures in the table.

Table 2 was checked by using it to calculate

$$
\int_{y_{i-1}}^{y_{i}} J_{1}(x) d x=\left[-J_{0}(x)\right]_{y_{i-1}}^{y_{i}}=J_{0}\left(y_{i-1}\right)-J_{0}\left(y_{i}\right)
$$

over each half-cycle, where the $y_{i}$ are the zeros of $J_{1}(x)$. The results were calculated to ten places of decimals and in each case were accurate to within two units in the last place. 
TABLE 1

\begin{tabular}{|c|c|c|c|c|c|c|c|c|}
\hline & $x$ & & $J_{0}(x)$ & & $x$ & & $J_{0}(x)$ & \\
\hline & .00000 & 00000 & & & 5.52007 & 81103 & & \\
\hline 1 & .01274 & 44512 & . & 1 & 5.53668 & 49893 & .00564 & 199 \\
\hline 2 & .06664 & 37005 & . 9988899624 & 2 & 5.60691 & 93443 & .02928 & \\
\hline 3 & .16156 & 67593 & . 9934846849 & 3 & 5.73061 & 04883 & .06978 & 6215 \\
\hline 4 & .29410 & 48650 & . $97849 \quad 22054$ & 4 & & 65740 & .12301 & \\
\hline 5 & .45947 & 04869 & . 9479140344 & 5 & & 91260 & .18206 & 2481 \\
\hline 6 & .65168 & 75524 & . 8966110444 & 6 & 6.36927 & 09191 & .23760 & 1437 \\
\hline 7 & .86380 & & . 8219796824 & 7 & & & .27944 & 5548 \\
\hline 8 & 1.08816 & 852 & $.72517 \quad 2985$ & 8 & & & .29921 & \\
\hline 9 & 31665 & & . 6113606080 & 9 & & 09779 & .29294 & 4122 \\
\hline 0 & 1.54101 & & . & 10 & & & .26243 & 8001 \\
\hline 1 & 1.753 & & $.36721 \quad 18$ & & & & .21449 & 2687 \\
\hline 2 & 1.94535 & 50709 & . & 12 & & 685 & .15853 & 5815 \\
\hline 3 & 2.11072 & & .1605205 & 3 & & 94 & .10381 & 9197 \\
\hline 14 & 2.24325 & & $.08645 \quad 02$ & 4 & & & .05742 & \\
\hline 5 & 2.33818 & 18573 & .03506038 & 15 & & & .02366 & 256 \\
\hline 16 & 2.39208 & 11065 & . 0066336641 & 16 & & 10339 & .00451 & 209 \\
\hline & 2.40482 & & & & & & & \\
\hline 1 & 2.42133 & & .0085411 & 1 & & & .00450 & \\
\hline 2 & 2.49115 & 69581 & .0439783230 & 2 & & 43400 & -.02345 & 7128 \\
\hline 3 & 2.61412 & 19276 & -.1034259686 & 3 & & 95579 & -.05612 & 1538 \\
\hline 4 & 2.785 & & -.17919070 & 4 & & & -.09944 & 2036 \\
\hline 5 & 3.000 & & -.2600626 & 5 & & & -.14809 & \\
\hline 6 & 3.24903 & 28661 & -.3325174168 & 6 & & 71589 & -.19457 & 9681 \\
\hline 7 & 2381 & 87438 & -.3832809269 & 7 & & 24462 & -.23045 & 1207 \\
\hline 8 & & & -.40269939 & 8 & & & -.24845 & 5719 \\
\hline 9 & 4. & 58 & $\begin{array}{lll}-.38757 & 11\end{array}$ & 9 & & & -.24484 & \\
\hline 10 & 4.40108 & & -.3420366 & 0 & & & -.22066 & 4619 \\
\hline 11 & 4.67587 & & $-.27600 \quad 293$ & 11 & & 51931 & -.18130 & 8226 \\
\hline 12 & & & -.20186704 & 12 & & & -.13461 & 5167 \\
\hline 3 & & & -.1311013 & & & & -.08848 & 0014 \\
\hline 14 & 1078 & & -.0720725 & & & & -.04908 & \\
\hline 15 & 5.43374 & & -.0295709059 & 15 & 11.70457 & 80120 & -.02026 & 32192 \\
\hline & $\begin{array}{l}5.50356 \\
5.52007\end{array}$ & $\begin{array}{l}87282 \\
81103\end{array}$ & -.0056257234 & 16 & 11.77490 & 55314 & -.00386 & 8102 \\
\hline
\end{tabular}


TABLE 1-Continued

$x$

11.7915344391

$1 \quad 11.80817 \quad 17029$

$2 \quad 11.87853 \quad 45618$

$3 \quad 12.0024520168$

$\begin{array}{llll}4 & 12.17547 & 40932\end{array}$

$\begin{array}{llll}5 & 12.39135 & 09018\end{array}$

$\begin{array}{llll}6 & 12.64228 & 09694\end{array}$

$\begin{array}{llll}7 & 12.91919 & 53359\end{array}$

$\begin{array}{llll}8 & 13.21208 & 57321\end{array}$

$9 \quad 13.51036 \quad 64157$

$10 \quad 13.80325 \quad 68119$

$11 \quad 14.08017 \quad 11784$

$12 \quad 14.3311012460$

$\begin{array}{lllll}13 & 14.54697 & 80546\end{array}$

$14 \quad 14.72000 \quad 01310$

$\begin{array}{llll}15 & 14.84391 & 75860\end{array}$

$\begin{array}{lll}16 & 14.91428 & 04449\end{array}$ 14.9309177086

$1 \quad 14.9475590159$

$2 \quad 15.0179389756$

$\begin{array}{llll}3 & 15.14188 & 65473\end{array}$

$\begin{array}{llll}4 & 15.31495 & 06747\end{array}$

$\begin{array}{llll}5 & 15.53087 & 99495\end{array}$

$6 \quad 15.78187 \quad 10027$

$\begin{array}{lll}7 & 16.05885 & 26700\end{array}$

$\begin{array}{llll}8 & 16.35181 & 42497\end{array}$

$\begin{array}{llll}9 & 16.6501674269\end{array}$

$10 \quad 16.9431290066$

$11 \quad 17.22011 \quad 06739$

$\begin{array}{llll}12 & 17.47110 & 17271\end{array}$

$\begin{array}{lll}13 & 17.6870310019\end{array}$

$\begin{array}{lll}14 & 17.86009 & 51293\end{array}$

$\begin{array}{lll}15 & 17.98404 & 27010\end{array}$

$\begin{array}{lll}16 & 18.05442 & 26607\end{array}$ 18.0710639679

$$
J_{0}(x)
$$

.0038645915

.0201243811

.0482370070

.0856863326

.1279926729

.1687386824

.2005559550

.2169970655

.2145833409

.1940142035

.1598741093

.1190000109

.0783794720

.0435503186

.0179993958

.0034381248

$-.0034351301$

$-.0178991458$

$-.0429496191$

$-.0764067698$

$-.1143361880$

$-.1510388400$

$-.1799039278$

$-.1950768842$

$-.1933188429$

$-.1751401558$

$-.1445858966$

$-.1077931239$

$-.0710930831$

$-.0395430642$

$-.0163551985$

$-.0031253477$ $x$

18.0710639679

$1 \quad 18.0877075348$

$2 \quad 18.1580970515$

$3 \quad 18.28206 \quad 14541$

$4 \quad 18.4551490820$

$\begin{array}{llll}5 & 18.67110 & 76781\end{array}$

$6 \quad 18.92213 \quad 28135$

$\begin{array}{lll}7 & 19.19915 & 20923\end{array}$

$8 \quad 19.49215 \quad 34535$

$9 \begin{array}{lll}9 & 19.79054 & 71443\end{array}$

$\begin{array}{ll}10 & 20.08354 \quad 85055\end{array}$

$11 \quad 20.36056 \quad 77843$

$\begin{array}{llll}12 & 20.61159 & 29197\end{array}$

$13 \quad 20.82755 \quad 15158$

$\begin{array}{lll}14 & 21.00063 & 91437\end{array}$

$\begin{array}{llll}15 & 21.12460 & 35463\end{array}$

$\begin{array}{lll}16 & 21.19499 & 30630\end{array}$ 21.2116366299

$1 \quad 21.22828 \quad 15866$

$\begin{array}{llll}2 & 21.29867 & 69809\end{array}$

$3 \quad 21.42265 \quad 17345$

$\begin{array}{llll}4 & 21.59575 & 38153\end{array}$

$5 \quad 21.81173 \quad 04440$

$\begin{array}{llll}6 & 22.06277 & 65401\end{array}$

$\begin{array}{llll}7 & 22.33981 & 89501\end{array}$

$8 \quad 22.63284 \quad 47770$

$9 \quad 22.93126 \quad 33838$

$\begin{array}{lll}10 & 23.22428 & 92107\end{array}$

$\begin{array}{llll}11 & 23.50133 & 16207\end{array}$

$\begin{array}{llll}12 & 23.75237 & 77168\end{array}$

$\begin{array}{llll}13 & 23.96835 & 43455\end{array}$

$\begin{array}{lll}14 & 24.14145 & 64263\end{array}$

$\begin{array}{llll}15 & 24.26543 & 11799\end{array}$

$\begin{array}{llll}16 & 24.33582 & 65742\end{array}$ $24.35247 \quad 15308$

$$
J_{0}(x)
$$

.0031228948

.0162788282

.0390892889

.0696069216

.1042843725

.1379452734

.1645442527

.1786844763

.1773310142

.1608771698

.1329788950

.0992499767

.0655196366

.0364696948

.0150918172

.0028847612

$-.0028827393$

$-.0150312702$

$-.0361116663$

$-.0643488934$

$-.0964881035$

$-.1277547593$

$-.1525457750$

$-.1658305446$

$-.1647472215$

$-.1496108067$

$-.1237803876$

$-.0924599613$

$-.0610791462$

$-.0340163997$

$-.0140819757$

$-.0026923112$ 
TABLE 1-Continued

$$
x \quad J_{0}(x)
$$

24.3524715308

$\begin{array}{llll}1 & 24.3691174027\end{array}$

$\begin{array}{llll}2 & 24.43951 & 66677\end{array}$

$\begin{array}{llll}3 & 24.56349 & 82381\end{array}$

$\begin{array}{llll}4 & 24.73660 & 98370\end{array}$

$\begin{array}{llll}5 & 24.95259 & 83413\end{array}$

$\begin{array}{lll}6 & 25.20365 & 82413\end{array}$

$\begin{array}{llll}7 & 25.48071 & 58846\end{array}$

$\begin{array}{llll}8 & 25.77375 & 78237\end{array}$

$\begin{array}{llll}9 & 26.07219 & 28391\end{array}$

$\begin{array}{lll}10 & 26.36523 & 47782\end{array}$

$\begin{array}{lll}11 & 26.64229 & 24215\end{array}$

$\begin{array}{lll}12 & 26.89335 & 23215\end{array}$

$\begin{array}{llll}13 & 27.10934 & 08258\end{array}$

$\begin{array}{llll}14 & 27.28245 & 24247\end{array}$

$\begin{array}{lll}15 & 27.40643 & 39951\end{array}$

$\begin{array}{lll}16 & 27.47683 \quad 32601\end{array}$ 27.4934791320

$\begin{array}{lllll}1 & 27.51012 & 56384 & -.00253 & 24019\end{array}$

$\begin{array}{lllll}2 & 27.58052 & 75870 & -.01320 & 95154\end{array}$

$\begin{array}{lllll}3 & 27.70451 & 38837 & -.03175 & 59526\end{array}$

$\begin{array}{lllll}4 & 27.87763 & 20816 & -.0566387732\end{array}$

$\begin{array}{llll}5 & 28.09362 & 88193\end{array}$

$\begin{array}{llll}6 & 28.34469 & 82897\end{array}$

$\begin{array}{llll}7 & 28.62176 & 64945\end{array}$

$\begin{array}{llll}8 & 28.91481 & 96043\end{array}$

$\begin{array}{lll}9 & 29.21326 & 59961\end{array}$

$10 \quad 29.5063191059$

$\begin{array}{llll}11 & 29.78338 & 73107\end{array}$

$12 \quad 30.03445 \quad 67811$

$\begin{array}{llll}13 & 30.25045 & 35188\end{array}$

$\begin{array}{llll}14 & 30.42357 & 17167\end{array}$

$\begin{array}{lll}15 & 30.54755 \quad 80134\end{array}$

$16 \quad 30.6179599620$ 30.6346064684

.0026906195

.0140325020

.0337247328

.0601263461

.0902133604

.1195324224

.1428384354

.1554020499

.1545096015

.1404206959

.1162587219

.0868957273

.0574336567

.0319994405

.0132509005

.0025338391

$-.0850221095$

$-.1127168644$

$-.1347753040$

$-.1467213774$

$-.1459696665$

$-.1327389002$

$-.1099596619$

$-.0822281150$

$-.0543712708$

$-.0303032039$

$-.0125514239$

$-.0024004013$ $x$

$J_{0}(x)$

\begin{tabular}{|c|c|c|}
\hline & $x$ & $J_{0}(x)$ \\
\hline & & \\
\hline 1 & $30.65125 \quad 34327$ & .0023991633 \\
\hline 2 & 30.7216573180 & .0125161523 \\
\hline 3 & 30.8456470254 & .0300959796 \\
\hline 4 & 31.0187699856 & .0536950891 \\
\hline 5 & 31.2347726651 & .0806346686 \\
\hline 6 & 31.4858490422 & .1069479499 \\
\hline 7 & 31.7629248688 & .1279392673 \\
\hline 8 & 32.0559860401 & 13934 93292 \\
\hline 9 & $32.35444 \quad 06419$ & .1387048969 \\
\hline 10 & $32.64750 \quad 18132$ & .1261935228 \\
\hline 11 & $32.92457 \quad 76398$ & .1045843845 \\
\hline 12 & 33.1756540169 & .0782396934 \\
\hline 13 & 33.3916566964 & .0517515312 \\
\hline 14 & 33.5647796566 & .0288508462 \\
\hline 15 & 33.6887693640 & .0119521353 \\
\hline 16 & 1732 & .0022860352 \\
\hline & 5820213 & \\
\hline 1 & 33.7924675193 & -.0022849556 \\
\hline 2 & 33.8628728479 & -.0119216140 \\
\hline 3 & 33.9868650970 & -.0286717157 \\
\hline 4 & 34.1599916062 & -.0511672201 \\
\hline 5 & 34.3759987138 & -.0768629839 \\
\hline 6 & 34.6270802379 & -.1019826451 \\
\hline 7 & 11617446 & -.1220477212 \\
\hline 8 & 35.1972289237 & -.1329871140 \\
\hline 9 & 35.4956896437 & -.1324266679 \\
\hline 10 & 35.7887568228 & -.1205295168 \\
\hline 11 & 36.0658383295 & -.0999272078 \\
\hline 12 & 36.3169198536 & -.0747803172 \\
\hline 13 & 36.5329269612 & -.0494771799 \\
\hline 14 & 36.7060534704 & -.0275890427 \\
\hline 15 & 36.8300457195 & -.0114312041 \\
\hline 16 & 36.9004510481 & -.0021865932 \\
\hline & 36.9170983537 & \\
\hline
\end{tabular}

.0023991633

.0125161523

.0300959796

.0536950891

.0806346686

.1069479499

.1279392673

.1393493292

.1387048969

.1261935228

.1045843845

.0782396934

.0517515312

.0288508462

.0119521353

.0022860352

$-.0022849556$

$-.0286717157$

$-.0511672201$

.0768629839

$-.1019826451$

$-.1220477212$

$-.1329871140$

66679

$-.0999272078$

$-.0747803172$

$-.0275890427$

36.9170983537 
TABLE 1-Continued

\begin{tabular}{rrrrr}
\multicolumn{4}{c}{$x$} & \multicolumn{2}{c}{$J_{0}(x)$} \\
& 36.91709 & 83537 & & \\
1 & 36.93374 & 59205 & .00218 & 56417 \\
2 & 37.00415 & 23534 & .01140 & 44585 \\
3 & 37.12814 & 65473 & .02743 & 21950 \\
4 & 37.30127 & 57720 & .04896 & 56861 \\
5 & 37.51728 & 62677 & .07357 & 53320 \\
6 & 37.76837 & 17300 & .09765 & 02107 \\
7 & 38.04545 & 75826 & .11690 & 15020 \\
8 & 38.33852 & 93584 & .12742 & 34635 \\
9 & 38.63699 & 47599 & .12693 & 02235 \\
10 & 38.93006 & 65358 & .11556 & 53707 \\
11 & 39.20715 & 23884 & .09584 & 13189 \\
12 & 39.45823 & 78507 & .07174 & 25318 \\
13 & 39.67424 & 83464 & .04747 & 84574 \\
14 & 39.84737 & 75711 & .02647 & 94775 \\
15 & 39.97137 & 17650 & .01097 & 29247 \\
16 & 40.04177 & 81979 & .00209 & 90896 \\
& 40.05842 & 57646 & & \\
1 & 40.07507 & 35355 & -.00209 & 82432 \\
2 & 40.14548 & 08322 & -.01094 & 92382 \\
3 & 40.26947 & 65473 & -.02634 & 06534 \\
4 & 40.44260 & 78959 & -.04702 & 58304 \\
5 & 40.65862 & 10416 & -.07067 & 63342 \\
6 & 40.90970 & 95842 & -.09382 & 67282 \\
7 & 41.18679 & 88362 & -.11235 & 56730 \\
8 & 41.47987 & 42074 & -.12250 & 42137 \\
9 & 41.77834 & 32704 & -.12206 & 57451 \\
10 & 42.07141 & 86416 & -.11116 & 79231 \\
11 & 42.34850 & 78936 & -.09221 & 87488 \\
12 & 42.59959 & 64362 & -.06904 & 71411 \\
13 & 42.81560 & 95819 & -.04570 & 38541 \\
14 & 42.98874 & 09305 & -.02549 & 38175 \\
15 & 43.11273 & 66456 & -.01056 & 56711 \\
16 & 43.18314 & 39423 & -.00202 & 13118 \\
& 43.19979 & 17132 & & \\
& & & &
\end{tabular}

$x$

$J_{0}(x)$

43.1997917132

$\begin{array}{llllll}1 & 43.2164396470 \quad .00202 & 05537\end{array}$

$\begin{array}{lllll}2 & 43.28684 & 76319 & .01054 & 45035\end{array}$

$\begin{array}{lllll}3 & 43.41084 & 45592 & .0253698152\end{array}$

$\begin{array}{llllll}4 & 43.58397 & 76004 & .04529 & 96158\end{array}$

$\begin{array}{lllll}5 & 43.79999 & 28578 & .06809 & 50035\end{array}$

$\begin{array}{lllll}6 & 44.05108 & 38550 \quad .09041 & 97790\end{array}$

$\begin{array}{lllll}7 & 44.32817 & 58158 & .10830 & 19105\end{array}$

$\begin{array}{llllll}8 & 44.62125 & 40521 & .11811 & 38815\end{array}$

$\begin{array}{llllll}9 & 44.91972 & 60329 & .11772 & 07481\end{array}$

$\begin{array}{llllll}10 & 45.21280 & 42692 & .10723 & 69760\end{array}$

$\begin{array}{llllll}11 & 45.48989 & 62300 & .08897 & 80881\end{array}$

$\begin{array}{llllll}12 & 45.74098 & 72272 & .06663 & 43209\end{array}$

$\begin{array}{llllll}13 & 45.95700 & 24846 \quad .04411 & 43990\end{array}$

$\begin{array}{llllllll}14 & 46.13013 & 55258 & .02461 & 06003\end{array}$

$\begin{array}{llllll}15 & 46.25413 & 24531 & .01020 & 06291\end{array}$

$\begin{array}{lll}16 & 46.32454 & 04380\end{array}$

$46.34118 \quad 83717$

.0019515847

$\begin{array}{lllll}1 & 46.35783 & 64373 & -.00195 & 08992\end{array}$

$2 \quad 46.42824 \quad 49795 \quad-.01018 \quad 15645$

$\begin{array}{lllll}3 & 46.55224 & 28884 & -.02449 & 89606\end{array}$

$\begin{array}{lllll}4 & 46.72537 & 73001 & -.0437505072\end{array}$

$\begin{array}{lllll}5 & 46.94139 & 42674 & -.06577 & 72703\end{array}$

$\begin{array}{lllll}6 & 47.19248 & 72523 & -.0873588503\end{array}$

$\begin{array}{lllll}7 & 47.46958 & 14065 & -.10465 & 74117\end{array}$

$8 \quad 47.7626619629 \quad-.1141640209$

$9 \quad 48.0611363063 \quad-.1138089156$

$\begin{array}{llllll}10 & 48.35421 & 68627 & -.10369 & 54948\end{array}$

$\begin{array}{llllll}11 & 48.63131 & 10169 & -.08605 & 66385\end{array}$

$\begin{array}{llllll}12 & 48.88240 & 40018 & -.06445 & 79246\end{array}$

$\begin{array}{lllll}13 & 49.09842 & 09691 & -.04267 & 99914\end{array}$

$\begin{array}{llllll}14 & 49.27155 & 53808 & -.02381 & 32307\end{array}$

$\begin{array}{llllll}15 & 49.39555 & 32897 & -.00987 & 09780\end{array}$

$\begin{array}{lllll}16 & 49.46596 & 18319 & -.00188 \quad 86072\end{array}$ 49.4826098974 
TABLE 1-Continued

$x \quad J_{0}(x)$

49.4826098974

49.4992580712

249.5696670711

49.6936657858

49.8668013228

50.0828196942

50.3339143111

50.6110102663

50.9040927276

51.2025690110

$10 \quad 51.49565 \quad 14723$

$11 \quad 51.77274 \quad 74275$

$\begin{array}{llll}12 & 52.02384 & 20444\end{array}$

$\begin{array}{llll}13 & 52.23986 & 04158\end{array}$

$\begin{array}{llll}14 & 52.41299 & 59528\end{array}$

$\begin{array}{llll}15 & 52.53699 & 46675\end{array}$

$16 \quad 52.60740 \quad 36674$

52.6240518411

$1 \quad 52.64070 \quad 01048$

$2 \quad 52.71110 \quad 94851$

$3 \quad 52.83510 \quad 88697$

$4 \quad 53.00824 \quad 53420$

$5 \quad 53.22426 \quad 48803$

$6 \quad 53.47536 \quad 08536$

$\begin{array}{llll}7 & 53.75245 & 83057\end{array}$

$8 \quad 54.04554 \quad 23502$

$9 \begin{array}{lll}8 & 54.34402 & 02460\end{array}$

$10 \quad 54.63710 \quad 42905$

$11 \quad 54.9142017426$

$12 \quad 55.1652977159$

$13 \quad 55.3813172542$

$14 \quad 55.5544537265$

$15 \quad 55.67845 \quad 31111$

$16 \quad 55.74886 \quad 24914$ 55.7655107550
.0018879842

.0098536907

.0237120292

.0423501575

.0636811286

.0845890824

.1013576521

.1105855944

.1102627587

.1004831520

.0834052240

.0624817090

.0413769630

.0230886479

.0095713465

.0018313567

$-.0018307874$

$-.0095555769$

$-.0229963548$

$-.0410761891$

$-.0617733832$

$-.0820670662$

$-.0983515046$

$-.1073238205$

$-.1070286465$

$-.0975519386$

$-.0809846590$

$-.0606767598$

$-.0401864126$

$-.0224264133$

$-.0092974390$

$-.0017790152$
55.7821590943

$2 \quad 55.8525687941$

$3 \quad 55.97656 \quad 87414$

$4 \quad 56.14970 \quad 59995$

$5 \quad 56.36572 \quad 65182$

$6 \quad 56.61682 \quad 36311$

$\begin{array}{lll}7 & 56.89392 & 23408\end{array}$

$\begin{array}{lll}8 & 57.18700 & 77154\end{array}$

$\begin{array}{llll}9 & 57.48548 \quad 69658\end{array}$

$\begin{array}{llll}10 & 57.77857 & 23404\end{array}$

$\begin{array}{llll}11 & 58.05567 & 10501\end{array}$

$12 \quad 58.30676 \quad 81630$

$\begin{array}{llll}13 & 58.52278 & 86817\end{array}$

$\begin{array}{llll}14 & 58.69592 & 59398\end{array}$

$\begin{array}{llll}15 & 58.81992 & 58871\end{array}$

$16 \quad 58.89033 \quad 55869$

58.9069839261

$1 \quad 58.9236323295$

258.9940423003

$3 \quad 59.11804 \quad 27250$

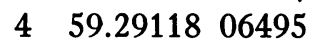

$\begin{array}{llll}5 & 59.50720 & 19998\end{array}$

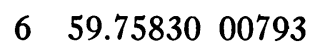

$\begin{array}{llll}7 & 60.03539 & 98556\end{array}$

$8 \quad 60.32848 \quad 63585$

$9 \quad 60.62696 \quad 67579$

$\begin{array}{lll}10 & 60.92005 & 32608\end{array}$

$12 \quad 61.4482511166$

$13 \quad 61.66427 \quad 24669$

14

15

16
$11 \quad 61.19715 \quad 30371$ $x$

55.7655107550

$$
J_{0}(x)
$$

.0017784922

.0092829778

.0223417 .954

.0399106704

.0600274072

.0797579441

.0955978634

.1043345986

.1040633501

.0948631094

.0787632823

.0590196906

.0390930391

.0218180708

.0090457744

.0017309189

$-.0017304364$

$-.0090324503$

$-.0217401207$

$-.0388390402$

$-.0584215607$

$-.0776333587$

$-.0930632526$

$-.1015819737$

$-.1013315847$

$-.0923850042$

$-.0767151960$

$-.0574913542$

$-.0380843044$

$61.8374103914-.0212566871$

$\begin{array}{lllll}61.96141 & 08161 & -.0088134966\end{array}$

$\begin{array}{lllll}62.03182 & 07869 & -.00168 & 65234\end{array}$

62.0484691902 
TABLE 2

\begin{tabular}{|c|c|c|c|c|c|}
\hline & $x$ & $J_{1}(x)$ & & $x$ & $J_{1}(x)$ \\
\hline & .0000000000 & & & 7.0155866698 & \\
\hline 1 & .0203062503 & .0101526018 & 1 & 7.0323219654 & .0050163154 \\
\hline 2 & .1061861074 & .0530182575 & 2 & 7.1030994235 & .0260688611 \\
\hline 3 & .2574308620 & .1276521143 & 3 & 7.2277470376 & .0622685746 \\
\hline 4 & 4686091943 & .2279316731 & 4 & 7.4017886125 & .1100966693 \\
\hline 5 & .73209 29378 & .3420647543 & 5 & 7.6189374325 & .1635480168 \\
\hline 6 & 1.0383601743 & .4522819201 & 6 & 7.8713460560 & .2143084782 \\
\hline 7 & $1.37634 \quad 19817$ & .5375852259 & 7 & 8.1498920857 & .2531194883 \\
\hline 8 & 1.7338229846 & .5794953889 & 8 & 8.4445082807 & .2721607618 \\
\hline 9 & 2.0978829856 & .5685114490 & 9 & $8.74454 \quad 65243$ & .2675221198 \\
\hline 10 & 2.4553639885 & $.50780 \quad 82279$ & 10 & 9.0391627193 & .2405322625 \\
\hline 11 & 2.7933457959 & .4119088809 & 11 & 9.3177087490 & .1972125700 \\
\hline 12 & 3.0996130324 & .3010717052 & 12 & 9.5701173725 & .1461517084 \\
\hline 13 & 3.3630967759 & .1945839510 & 13 & 9.7872661925 & .0959129504 \\
\hline 14 & 3.5742751082 & .10623 42640 & 14 & 9.9613077674 & .0531408898 \\
\hline 15 & 3.7255198628 & .0432954722 & 15 & 10.0859553815 & .0219191616 \\
\hline 16 & 3.8113997199 & .0081997562 & 16 & 10.1567328396 & .0041821325 \\
\hline & 3.8317059702 & & & 10.1734681351 & \\
\hline 1 & 3.8485790494 & -.0067805723 & 1 & 10.1901628485 & -.0041651430 \\
\hline 2 & 3.9199392273 & -.0350912559 & 2 & 10.2607686758 & -.0216790724 \\
\hline 3 & 4.0456130809 & -.0832373938 & 3 & 10.3851140273 & -.0519208365 \\
\hline 4 & $4.22108 \quad 75619$ & -.1458438197 & 4 & 10.5587335623 & -.0921285148 \\
\hline 5 & 4.4400241954 & -.2144193722 & 5 & 10.7753558100 & -.1374355137 \\
\hline 6 & 4.6945109307 & -.2779236119 & 6 & 11.0271523585 & -.1809268681 \\
\hline 7 & 4.9753502648 & -.3247141295 & 7 & 11.3050229316 & -.2147199134 \\
\hline 8 & $5.27239 \quad 20719$ & -.3455421188 & 8 & 11.5989247008 & -.2319738712 \\
\hline 9 & $5.57490 \quad 05681$ & -.3364108609 & 9 & 11.8982353706 & -.2290610599 \\
\hline 10 & $5.87194 \quad 23752$ & -.2998678712 & 10 & 12.1921371398 & -.2068224276 \\
\hline 11 & 6.1527817093 & -.24400 22677 & 11 & 12.4700077129 & -.1702173009 \\
\hline 12 & 6.4072684446 & -.1796591930 & 12 & $12.72180 \quad 42614$ & -.1265604959 \\
\hline 13 & 6.6262050781 & -.1172767942 & 13 & 12.9384265091 & -.0832824257 \\
\hline 14 & 6.8016795591 & -.0647102564 & 14 & 13.1120460441 & -.0462408298 \\
\hline 15 & $6.92735 \quad 34127$ & -.0266142650 & 15 & 13.2363913956 & -.0191014741 \\
\hline \multirow[t]{2}{*}{16} & 6.9987135906 & -.0050697404 & 16 & 13.3069972229 & -.0036475651 \\
\hline & 7.0155866698 & & & 13.3236919363 & \\
\hline
\end{tabular}


TABLES FOR EVALUATION OF INTEGRALS INVOLVING BESSEL FUNCTIONS 175

TABLE 2-Continued

\begin{tabular}{|c|c|c|c|c|c|c|}
\hline & $x$ & & $J_{1}(x)$ & & $x$ & $J_{1}(x)$ \\
\hline & 13.32369 & 19363 & & & 19.6158585105 & \\
\hline 1 & 13.34036 & 92371 & .0036392005 & 1 & 19.6325214378 & .0029989710 \\
\hline 2 & 13.41090 & 14225 & .0189570437 & 2 & 19.7029928337 & .0156352051 \\
\hline 3 & 13.53511 & 70816 & .0454656026 & 3 & 19.8271014354 & .0375537294 \\
\hline 4 & 13.70855 & 55312 & .0808285751 & 4 & 20.0003904040 & .0668970210 \\
\hline 5 & 13.92495 & 18418 & .1208559397 & 5 & 20.2166002095 & .1002696775 \\
\hline 6 & 14.17648 & 57662 & .1595088072 & 6 & 20.4679173449 & 1327031954 \\
\hline 7 & 14.45406 & 65200 & 1898134949 & 7 & 20.7452588608 & .15837 97464 \\
\hline 8 & 14.74766 & 17494 & .2056258827 & 8 & 21.0386010500 & .1720893345 \\
\hline 9 & 15.04666 & 02378 & .2035827840 & 9 & 21.3373418412 & .1708833480 \\
\hline 10 & 15.34025 & 54672 & .1842760601 & 10 & $21.63068 \quad 40304$ & .1551121291 \\
\hline 11 & 15.61783 & 62210 & .1520047172 & 11 & 21.9080255463 & .1282775447 \\
\hline 12 & 15.86937 & 01454 & .1132427689 & 12 & 22.1593426817 & .0957830326 \\
\hline 13 & 16.08576 & 64560 & .0746419546 & 13 & 22.3755524872 & .0632540801 \\
\hline 14 & 16.25920 & 49056 & .0414970116 & 14 & 22.5488414558 & .0352186914 \\
\hline 15 & 16.38342 & 05647 & .0171574777 & 15 & 22.6729500575 & $.01457 \quad 70470$ \\
\hline \multirow[t]{2}{*}{16} & 16.45395 & 27501 & .0032780206 & 16 & $22.74342 \quad 14534$ & .0027866763 \\
\hline & 16.47063 & 00509 & & & 22.7600843806 & \\
\hline 1 & 16.48729 & 82913 & -.0032729251 & 1 & 22.7767439258 & -.0027840720 \\
\hline 2 & 16.55779 & 21583 & -.0170576459 & 2 & 22.8472010186 & -.0145184126 \\
\hline 3 & 16.68194 & 03340 & -.0409459048 & 3 & $22.97128 \quad 44308$ & -.0348863684 \\
\hline 4 & 16.85528 & 45588 & -.0728803022 & 4 & 23.1445382281 & -.0621823121 \\
\hline 5 & 17.07156 & 33065 & -.1091292847 & 5 & 23.3607041509 & -.0932705856 \\
\hline 6 & 17.32296 & 05786 & -.1442661835 & 6 & 23.6119702781 & -.1235419341 \\
\hline 7 & 17.60039 & 05295 & -.1719726270 & 7 & 23.8892555038 & -.1475767318 \\
\hline 8 & 17.89382 & 62558 & -.1866279783 & 8 & 24.1825381552 & -.1604977977 \\
\hline 9 & 18.19266 & 23056 & -.1850940367 & 9 & 24.4812183130 & -.1595175470 \\
\hline 10 & 18.48609 & 80319 & -.1678159539 & 10 & $24.77450 \quad 09644$ & -.1449209337 \\
\hline 11 & 18.76352 & 79828 & -.1386352301 & 11 & 25.0517861901 & -.1199454048 \\
\hline 12 & 19.01492 & 52549 & -.1034191552 & 12 & 25.3030523173 & -.0896251276 \\
\hline 13 & 19.23120 & 40026 & -.0682425400 & 13 & 25.5192182401 & -.0592229479 \\
\hline 14 & 19.40454 & 82274 & -.0379723479 & 14 & $25.69247 \quad 20374$ & -.0329898365 \\
\hline 15 & 19.52869 & 64031 & -.0157098187 & 15 & 25.8165554496 & -.0136590977 \\
\hline \multirow[t]{2}{*}{16} & 19.59919 & 02701 & -.0030024768 & 16 & 25.8870125424 & -.0026116864 \\
\hline & 19.61585 & 85105 & & & 25.9036720876 & \\
\hline
\end{tabular}


TABLE 2-Continued

$x$

25.9036720876

$1 \quad 25.9203293474$

$\begin{array}{llll}2 & 25.99077 & 67744\end{array}$

$\begin{array}{lll}3 & 26.11484 & 31639\end{array}$

$\begin{array}{llll}4 & 26.28807 & 31930\end{array}$

$5 \quad 26.5042094607$

$6 \quad 26.75544 \quad 11174$

$7 \quad 27.03268 \quad 83032$

$\begin{array}{lll}8 & 27.32593 & 07199\end{array}$

$\begin{array}{lll}9 & 27.62456 & 99027\end{array}$

$10 \quad 27.9178123194$

$11 \quad 28.19505 \quad 95052$

$12 \quad 28.44629 \quad 11619$

$13 \quad 28.6624274296$

$\begin{array}{llll}14 & 28.83565 & 74587\end{array}$

$\begin{array}{lll}15 & 28.95972 & 38482\end{array}$

$\begin{array}{lll}16 & 29.03017 & 12752\end{array}$ 29.0468285349

$\begin{array}{llll}1 & 29.06348 & 41780\end{array}$

$\begin{array}{lll}2 & 29.13392 & 47675\end{array}$

$\begin{array}{lll}3 & 29.25797 & 91152\end{array}$

$4 \quad 29.4311923308$

$5 \quad 29.6473076205$

$6 \quad 29.89851 \quad 48930$

$7 \quad 30.17573 \quad 51694$

$8 \quad 30.4689491244$

$9 \quad 30.7675593216$

$10 \quad 31.06077 \quad 32766$

$11 \quad 31.33799 \quad 35530$

$\begin{array}{llll}12 & 31.58920 & 08255\end{array}$

$13 \quad 31.8053161152$

$\begin{array}{lll}14 & 31.97852 & 93308\end{array}$

$\begin{array}{llll}15 & 32.10258 & 36785\end{array}$

$16 \quad 32.17302 \quad 42680$ 32.1896799110

$$
J_{1}(x)
$$

.0026096493

.0136113779

.0327175400

.0583428100

.0875598843

.1160508696

.1387226066

.1509745446

.1501574510

.1365089575

.1130533742

.0845219696

.0558769690

.0311374830

.0128955290

.0024660528

$-.0024643999$

$-.0128556731$

$-.0309089818$

$-.0551372476$

$-.0827850383$

$-.1097768854$

$-.1312935471$

$-.1429690393$

$-.1422743786$

$-.1294116541$

$-.1072287815$

$-.0802027911$

$-.0530414604$

$-.0295661656$

$-.0122473498$

$-.0023423778$ $x$

$$
J_{1}(x)
$$

32.1896799110

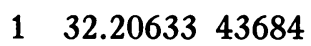

$2 \quad 32.27676 \quad 99432$

$\begin{array}{llll}3 & 32.40081 & 54597\end{array}$

$\begin{array}{llll}4 & 32.57401 & 63444\end{array}$

$5 \quad 32.7901162490$

$6 \quad 33.04130 \quad 56382$

$7 \quad 33.31850 \quad 61796$

$8 \quad 33.6116992609$

$\begin{array}{lll}9 & 33.91028 & 82003\end{array}$

$\begin{array}{llll}10 & 34.20348 & 12816\end{array}$

$\begin{array}{llll}11 & 34.48068 & 18230\end{array}$

$\begin{array}{llll}12 & 34.73187 & 12122\end{array}$

$\begin{array}{llll}13 & 34.94797 & 11168\end{array}$

$\begin{array}{lll}14 & 35.12117 & 20015\end{array}$

$\begin{array}{lll}15 & 35.24521 & 75180\end{array}$

$16 \quad 35.31565 \quad 30928$ 35.3323075501

$\begin{array}{lllll}1 & 35.34896 & 11121 & -.00223 & 44653\end{array}$

$2 \quad 35.4193929002-.0116587150$

$3 \quad 35.5434317477 \quad-.0280416792$

$\begin{array}{lllll}4 & 35.71662 & 33207 & -.05004 & 84236\end{array}$

$\begin{array}{lllll}5 & 35.93271 & 16074 & -.07519 & 26901\end{array}$

$\begin{array}{llllll}6 & 36.18388 & 74921 & -.09978 & 23087\end{array}$

$\begin{array}{llllll}7 & 36.46107 & 31306 & -.11943 & 51446\end{array}$

$8 \quad 36.7542504492 \quad-.1301638763$

$\begin{array}{llllll}9 & 37.05282 & 33358 & -.12963 & 88064\end{array}$

$\begin{array}{lllll}10 & 37.34600 & 06544 & -.11801 & 27490\end{array}$

$\begin{array}{llllll}11 & 37.62318 & 62929 & -.09785 & 65260\end{array}$

$12 \quad 37.87436 \quad 21776 \quad-.0732413135$

$\begin{array}{lllll}13 & 38.09045 & 04643 & -.04846 & 48482\end{array}$

$\begin{array}{llllll}14 & 38.26364 & 20373 & -.02702 & 71631\end{array}$

$\begin{array}{lllll}15 & 38.38768 & 08848 & -.01119 & 91603\end{array}$

$\begin{array}{lllll}16 & 38.45811 & 26729 & -.00214 & 22896\end{array}$ $38.47476 \quad 62348$ 
TABLE 2-Continued

$x$

$38.47476 \quad 62348$

$1 \quad 38.4914191040$

$2 \quad 38.5618479626$

$\begin{array}{llll}3 & 38.68588 & 16508\end{array}$

$\begin{array}{llll}4 & 38.85906 & 60202\end{array}$

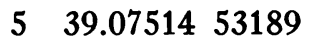

$6 \quad 39.3263107563$

$\begin{array}{llll}7 & 39.60348 & 48656\end{array}$

$8 \quad 39.8966499899$

$\begin{array}{lll}9 & 40.19521 & 04577\end{array}$

$10 \quad 40.48837 \quad 55820$

$11 \quad 40.7655496913$

$12 \quad 41.01671 \quad 51287$

$13 \quad 41.23279 \quad 44274$
41.4059787968

41.5300124850

41.6004413436

41.6170942128

41.6337465353

41.7041730810

41.8282026960

42.0013813780

42.2174535808

42.4686107699

42.7457757769

43.0389312737

43.3374819369

43.6306374337

43.9078024407

44.1589596298

44.3750318326

44.5482105146

44.6722401296

44.7426666753

44.7593189977

$$
J_{1}(x)
$$

.0021412735

.0111733773

.0268781611

.0479812388

.0721044757

.0957108701

.1145965682

.1249301313

.1244656086

.1133382116

.0940072264

.0703782521

.0465804345

.0259807709

.0107668903

.0020597434

$-.0020588518$

$-.0107440331$

$-.0258484372$

$-.0461508277$

$-.0693681412$

$-.0921005548$

$-.1103023920$

$-.1202811784$

$-.1198663996$

$-.1091788005$

$-.0905793974$

$-.0678268662$

$-.0449001454$

$-.0250472789$

$-.0103811283$

$-.0019860641$

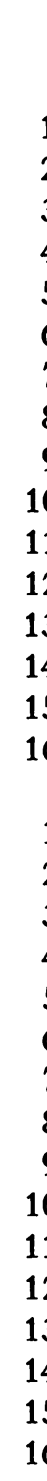

44.7593189977

$1 \quad 44.77597 \quad 08809$

$2 \quad 44.8463955686$

$3 \quad 44.9704219116$

$4 \quad 45.1435960250$

$5 \quad 45.3596625275$

$6 \quad 45.6108130909$

$\begin{array}{llll}7 & 45.88797 & 07859\end{array}$

$8 \quad 46.18111 \quad 85490$

$9 \quad 46.47966 \quad 13360$

$10 \quad 46.7728090991$

1147.0499667941

$12 \quad 47.30111 \quad 73575$

$\begin{array}{lll}13 & 47.51718 \quad 38600\end{array}$

$\begin{array}{llll}14 & 47.69035 & 79734\end{array}$

$15 \quad 47.81438 \quad 43164$

$\begin{array}{lll}16 & 47.88480 & 90041\end{array}$ 47.9014608872

$1 \quad 47.9181124121$

2. $47.98853 \quad 55848$

$3 \quad 48.1125592598$

$\begin{array}{lll}4 & 48.28572 & 96479\end{array}$

$\begin{array}{llll}5 & 48.50179 & 15025\end{array}$

$6 \quad 48.7529366631$

$\begin{array}{llll}7 & 49.03008 & 83960\end{array}$

$8 \quad 49.32322 \quad 98530$

$9 \quad 49.62176 \quad 62178$

$\begin{array}{llll}10 & 49.91490 & 76748\end{array}$

$\begin{array}{lll}11 & 50.19205 & 94077\end{array}$

$\begin{array}{llll}12 & 50.44320 & 45683\end{array}$

$13 \quad 50.65926 \quad 64229$

$14 \quad 50.83243 \quad 68110$

$\begin{array}{ll}15 & 50.9564604860\end{array}$

16

$$
J_{1}(x)
$$

.0019852730

.0103606792

.0249287731

.0445151717

.0669215430

.0888703803

.1064575916

.1161155971

.1157422770

.1054463168

.0875013044

.0655343925

.0433895839

.0242077320

.0100340865

.0019197690

$-.0019190607$

$-.0100156479$

$-.0241007718$

$-.0430420054$

$-.0647168956$

$-.0859579773$

$-.1029888602$

$-.1123550074$

$-.1120166719$

$-.1020723609$

$-.0847172272$

$-.0634597884$

$-.0420219605$

$-.0234473534$

$-.0097196893$

$51.0268836587-.0018597015$ 
TABLE 2-Continued

\begin{tabular}{|c|c|c|c|c|c|c|}
\hline & $x$ & $J_{1}(x)$ & & $x$ & & $J_{1}(x)$ \\
\hline & 51.0435351836 & & & 57.32752 & 54379 & \\
\hline 1 & 51.0601864126 & .0018590619 & 1 & 57.34417 & 62108 & .0017542236 \\
\hline 2 & 51.1306083339 & .0097029509 & 2 & 57.41459 & 62035 & .0091564627 \\
\hline 3 & 51.2546298048 & .0233501746 & 3 & 57.53861 & 42778 & .0220379680 \\
\hline 4 & 51.4277971154 & .0417060842 & 4 & 57.71177 & 68459 & .0393695712 \\
\hline 5 & 51.6438551303 & .0627167691 & 5 & 57.92782 & 89436 & .0592166524 \\
\hline 6 & 51.8949958277 & .0833144272 & 6 & 58.17896 & 27631 & $.07868 \quad 54349$ \\
\hline 7 & 52.1721426353 & .0998386147 & 7 & 58.45610 & 19805 & .0943185737 \\
\hline 8 & 52.4652788827 & .1089377477 & 8 & 58.74923 & 01998 & .1029455141 \\
\hline 9 & 52.7638099421 & 1086292546 & 9 & 59.04775 & 30834 & .1026850402 \\
\hline 10 & 53.0569461895 & .0990029593 & 10 & 59.34088 & 13027 & .0936130005 \\
\hline 11 & 53.3340929971 & .0821831360 & 11 & 59.61802 & 05201 & .0777302633 \\
\hline 12 & 53.5852336945 & .0615705817 & 12 & 59.86915 & 43396 & .0582489261 \\
\hline 13 & 53.8012917094 & .0407760577 & 13 & 60.08520 & 64373 & .0385843703 \\
\hline 14 & 53.9744590200 & .0227544296 & 14 & 60.25836 & 90054 & .0215350060 \\
\hline 15 & $54.09848 \quad 04909$ & .0094331183 & 15 & 60.38238 & 70797 & .0089286590 \\
\hline 16 & 54.1689024122 & .0018049432 & 16 & 60.45280 & 70724 & .0017085351 \\
\hline & 54.1855536411 & & & 60.46945 & 78453 & \\
\hline 1 & $54.20220 \quad 46228$ & -.0018043618 & 1 & 60.48610 & 84404 & -.0017080464 \\
\hline 2 & 54.2726254982 & -.0094178326 & 2 & 60.55652 & 76810 & -.0089157171 \\
\hline 3 & 54.3966451273 & -.0226656242 & 3 & 60.68054 & 44308 & -.0214597360 \\
\hline 4 & 54.5698098663 & -.0404873357 & 4 & 60.85370 & 51497 & -.0383395708 \\
\hline 5 & 54.7858646726 & -.0608913712 & 5 & 61.06975 & 49401 & -.0576729573 \\
\hline 6 & 55.0370016405 & -.0809007468 & 6 & 61.32088 & 60776 & -.0766427230 \\
\hline 7 & 55.3141443323 & -.0969609091 & 7 & 61.59802 & 23352 & -.0918811714 \\
\hline 8 & $55.60727 \quad 62264$ & -.1058145509 & 8 & 61.89114 & 74241 & -.1002979275 \\
\hline 9 & 55.9058028526 & -.1055317575 & 9 & 62.18966 & 71197 & -.1000569840 \\
\hline 10 & 56.1989347467 & -.0961948735 & 10 & 62.48279 & 22086 & -.0912285052 \\
\hline 11 & $56.47607 \quad 74385$ & -.0798637153 & 11 & 62.75992 & 84662 & -.0757591800 \\
\hline 12 & 56.7272144064 & -.0598406981 & 12 & 63.01105 & 96037 & -.0567778096 \\
\hline 13 & 56.9432692127 & -.0396348225 & 13 & 63.22710 & 93941 & -.0376132667 \\
\hline 14 & 57.1164339517 & -.0221195390 & 14 & 63.40027 & 01130 & -.0209945050 \\
\hline 15 & 57.2404535808 & -.0091704951 & 15 & 63.52428 & 68628 & -.0087050043 \\
\hline 16 & $57.31087 \quad 44562$ & -.0017547553 & 16 & 63.59470 & 61034 & -.0016657858 \\
\hline
\end{tabular}


TABLES FOR EVALUATION OF INTEGRALS INVOLVING BESSEL FUNCTIONS 179

Example of the Use of the Tables. The example

$$
\int_{0}^{\infty} J_{0}(x) d x=1
$$

given in $[1]$ is repeated here partly since an error in Watson ([6], p. 752) is revealed, and partly as an extension of the tabulation of

$$
\int_{x n}^{x_{n}+1} J_{0}(x) d x
$$

to a larger value of $\boldsymbol{n}$ than that obtainable from Watson ([6], p. 752).

Applying the integration coefficients directly to the values of $J_{0}(x)$ in Table 1 we get

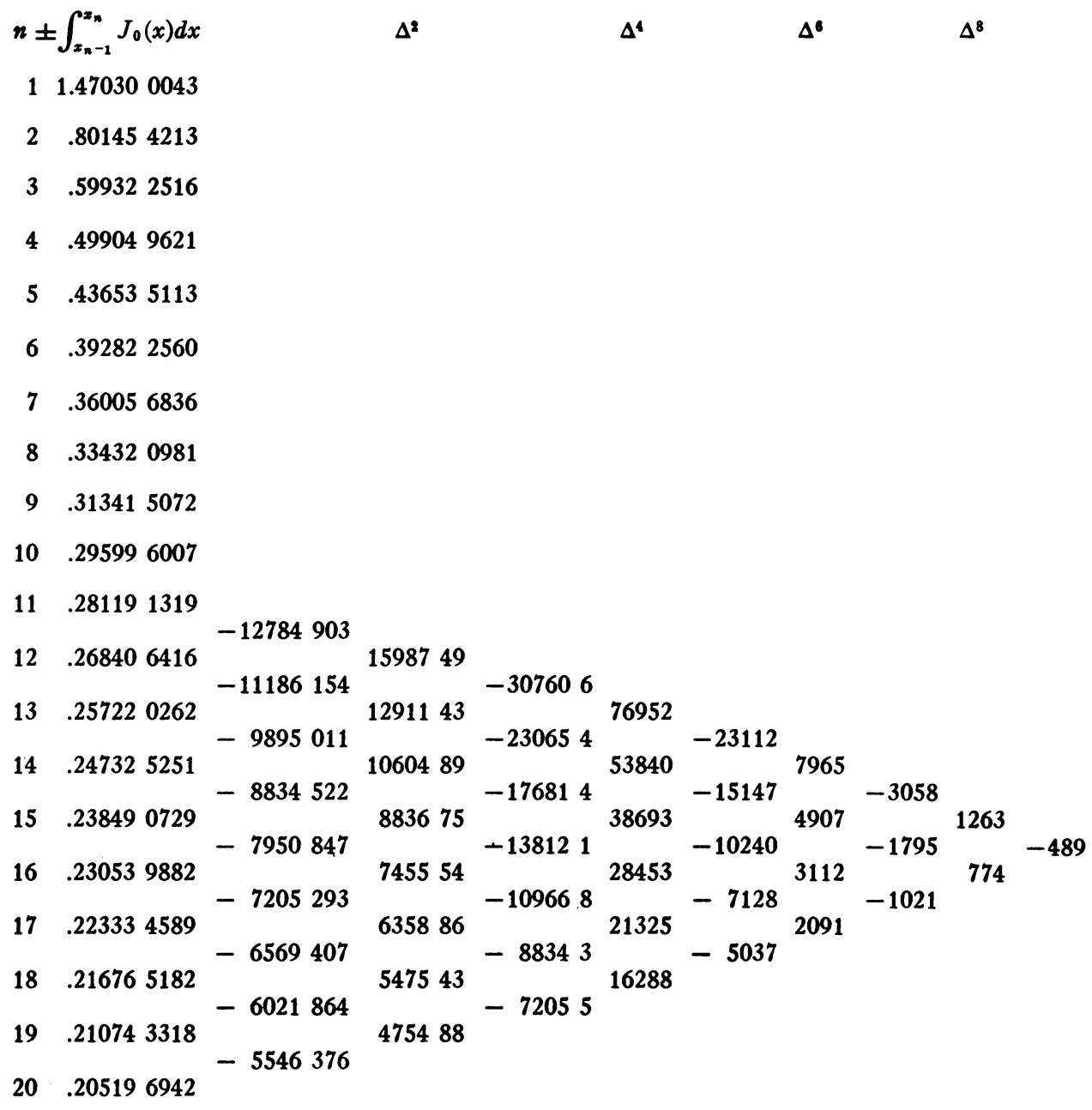


Now, choosing the first advancing row of differences we have (by Euler's transformation) approximately

$$
\begin{aligned}
\int_{0}^{\infty} J_{0}(x) d x= & 1.470300043-0.801454213+.599322516-.499049621 \\
& +.436535113-.392822560+.360056836-.334320981 \\
& +.313415072-.295996007+(1 / 2)(0.281191319) \\
& +(1 / 4)(0.012784903)+(1 / 8)(0.001598749) \\
& +(1 / 16)(0.000307606)+(1 / 32)(0.000076952) \\
& +(1 / 64)(0.000023112)+(1 / 128)(0.000007965) \\
& +(1 / 256)(0.000003058)+(1 / 512)(0.000001263) \\
& +(1 / 1024)(0.000000489)=0.999999992 .
\end{aligned}
$$

It should be noted that the first figure in the table

$$
1.4703000434=\int_{0}^{x_{1}} J_{0}(x) d x
$$

disagrees with the figure for this integral quoted in [1], where it is taken from Watson ([6], page 752). This integral was therefore calculated independently by interpolation,

$$
\int_{0}^{x+h} J_{0}(t) d t=\int_{0}^{x} J_{0}(t) d t+h J_{0}(x)-\frac{h^{2}}{2 !} J_{1}(x)+\frac{h^{3}}{3 !}\left(\frac{J_{1}(x)}{x}-J_{0}(x)\right),
$$

(Taylor's theorem) using tables of $J_{0}(x), J_{1}(x)$ [4] and a table [3] of $\int_{0}^{x} J_{0}(t) d t$. For this $x$ was taken to be 2.4 and $h$ to be 0.0048255577 , so that $x+h=2.4048255577$ is the first zero of $J_{0}(x)$. The result obtained agreed with (4) to ten decimal places, and this confirmation reveals an error in Watson? [6] where the value

$$
\frac{1}{2} \int_{0}^{x_{1}} J_{0}(x) d x=0.7352208
$$

is quoted. This value should be corrected to 0.7351500 .

Department of Applied Mathematics,

I. M. LoNGMAN

The Weizmann Institute of Science,

Rehovoth, Israel

1. I. M. Longman, "Note on a method for computing infinite integrals of oscillatory functions," Cambridge Phil. Soc., Proc., v. 52, 1956, p. 764.

2. T. J. I'A. Bromwich, An Introduction to the Theory of Infinite Series, The Macmillan Co., New York, 1942.

3. NBS Applied Mathematics Series, No. 37, Tables of Functions and of Zeros of Functions, U. S. Gov. Printing Office, Washington, D. C., 1954.

4. HARVARD UNIVERSITY, Computation Laboratory, Annals, v. 3 : Tables of the Bessel Functions of the First Kind of Orders Zero and One, Harvard University Press, Cambridge, Mass., 1947.

5. BRItish Association For The AdvanCEMENT OF SCIENCE, Committee on Mathematical Tables, Bessel Functions, v. 6, "Zeros of $J_{0}(x)$ and $J_{1}(x)$," Cambridge: at the University Press, 1950.

6. G. N. Watson, A Treatise on the Theory of Bessel Functions, Cambridge: at the University Press, 1948.

7. P. Davis \& P. Rabinowitz, "Abscissas and weights for Gaussian quadratures of high order," NBS Jn. of Research, v. 56, Research Paper 2645, 1956, p. 35-37. 